\title{
Measurement of sound, vibration and friction between soft materials under light loads ${ }^{\text {负 }}$
}

\author{
Adnan Akay ${ }^{\mathrm{a}, *}$, Blair Echols ${ }^{\mathrm{b}}$, Junqi Ding ${ }^{\mathrm{c}}$, Anne Dussaud $^{\mathrm{c}}$, Alex Lips $^{\mathrm{c}}$ \\ a Bilkent University, Mechanical Engineering Department, 06800 Bilkent, Ankara, Turkey \\ b Carnegie Mellon University, Mechanical Engineering Department, Pittsburgh, PA 15213, USA \\ ' Unilever Research \& Development, 40 Merritt Blvd. Trumbull, CT 06611, USA
}

\section{A R T I C L E I N F O}

\section{Article history:}

Received 25 May 2011

Received in revised form 5 November 2011

Accepted 14 December 2011

Available online 22 December 2011

\section{Keywords:}

Tactile

Soft materials

Friction measurement

\begin{abstract}
A B S T R A C T
Tactile perception of materials and surface texture involves friction under light normal loads and is fundamental to further advancing areas such as tactile sensing, haptic systems used in robotic gripping of sensitive objects, and characterization of products that range from fabrics to personal care products, such as lotions, on skin. This paper describes a new apparatus to measure friction simultaneously with dynamic quantities such as accelerations, forces, and sound pressures resulting from light sliding contact over a soft material, much like a finger lightly touching a soft material. The paper also introduces a novel friction and adhesion measurement method that can be particularly useful for soft materials and light normal loads.
\end{abstract}

(c) 2011 Elsevier B.V. All rights reserved.

\section{Introduction}

In recent years, haptic perception, one of the fundamental areas of cognitive engineering, has advanced with significant contributions from research in biology, engineering, and psychology. Although less well understood than audition and vision, the touch, or somatic sensation, continues to have an increasing significance in engineering and medical fields alike. For example, in the development of robotic grips or virtual reality hardware, tactile feedback is an essential element. In medicine, tactile sensitivity at fingertips is thought to have a role in diagnosis of and monitoring during rehabilitation for diabetes.

Haptic perception of materials and surfaces relies largely on touch, or tactile sensing, but also benefits from visual and auditory cues and, in some cases, from olfactory and even gustatory senses. Investigations have shown that visual or auditory cues can have a significant role in discriminating surface characteristics [viz., 1-4]. Studies on the role of multisensory integration of these signals on perception of surface finish or texture suggest that vision and touch

\footnotetext{
访 This research was carried out at Carnegie Mellon University.

* Corresponding author at: Bilkent University, Mechanical Engineering Department, 06800 Bilkent, Ankara, Turkey. Tel.: +90 3122903389 ; fax: +90312266 4126.

E-mail addresses: akay@bilkent.edu.tr, akay@cmu.edu (A. Akay).
}

act as independent sources [4] although in some cases vision can improve perception based on somatic sensation [5].

Multiple sensory inputs are utilized not only in judging or characterizing surface finish or its texture, as for fabrics and cosmetics, but also in identifying shape and weight of an object. In applications of haptic technologies to grasp and lift or to move very light-weight or fragile objects mechanically, tactile sensing has a primary role in the regulation of the applied power. In all such applications, there is a need to relate the physical characteristics of surface qualities and texture to somatic sensation and perception. However, the question remains open whether or not the subjective evaluations such as rough/smooth, sticky/slippery, and hard/soft can be identified reliably using objective measures. The extreme surface characteristics, of course, can be identified using a number of different methods. For surfaces with mixed or less pronounced physical characteristics, however, objective measures have not yet replaced human perceptions, and there continues to be a need to develop measurement techniques to emulate somatic sensation that also correlate well with perception.

The purpose of this paper is to describe a method to measure signals similar to the tactile signals that a finger senses when it lightly touches a soft surface while simultaneously capturing the air-borne acoustic signals generated due to the friction forces that develop between them. The method also includes a unique approach to measuring friction and adhesion between two surfaces that can pave the way to discern between each pair of the subjective qualities described above. 

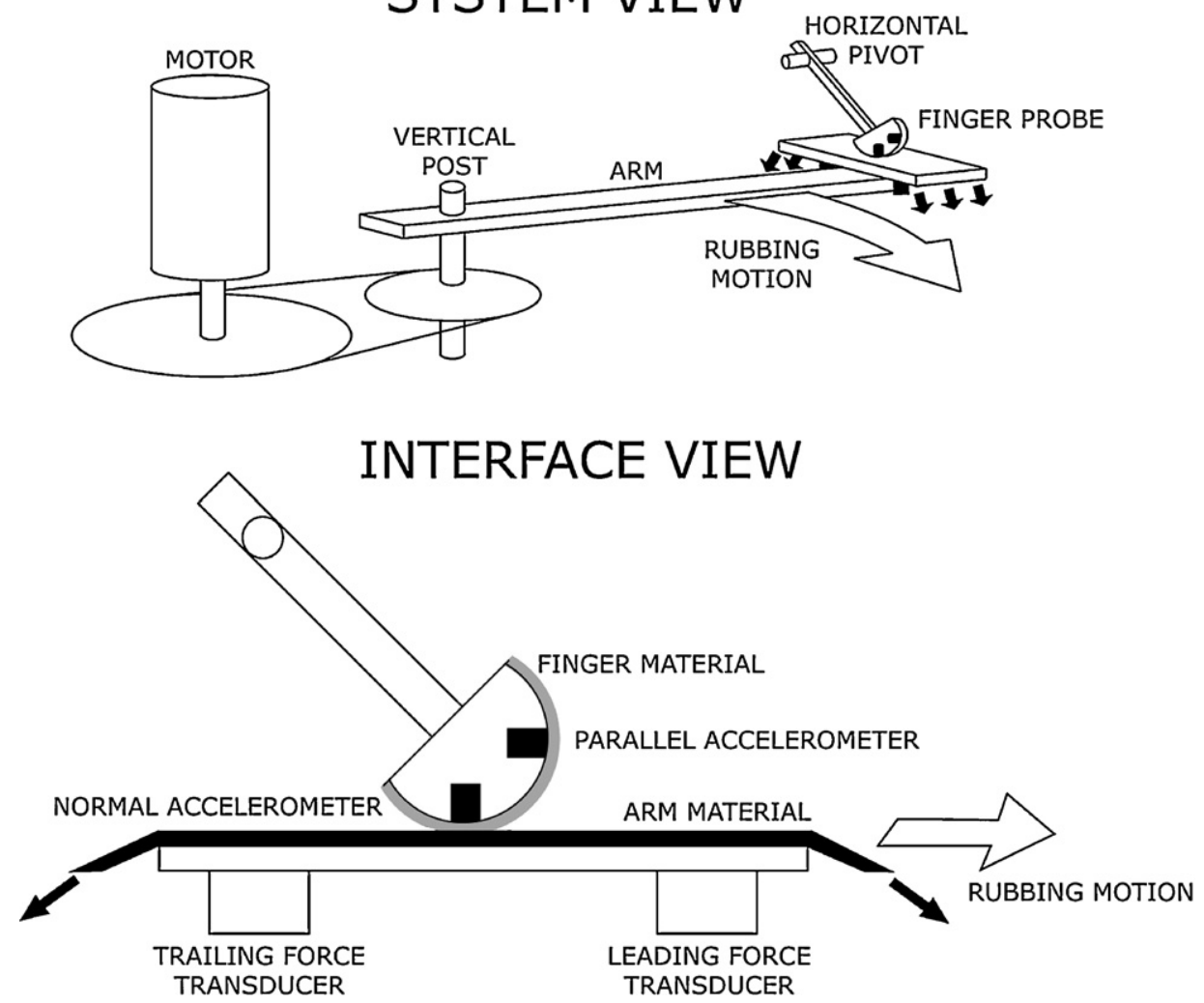

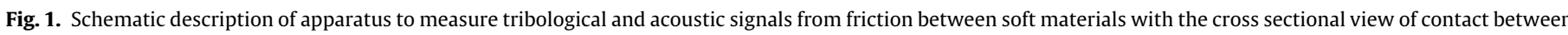
finger probe and arm during contact indicating the positions of accelerometers inside the finger probe and force transducers in the arm.

\section{Contact parameters between soft surfaces and their measurement}

The mechanical stimuli that form the basis of somatic sensory perception originate from contact forces that develop during the rubbing process, which contains information about smoothness, roughness, stickiness, slipperiness, hardness, and softness of the surfaces.

At room temperature, the physical parameters that are closely related to and influence subjective measures are the material properties, surface texture and shape, and adhesion between the surfaces. The sliding speed and contact pressure define the dynamic effects of these parameters. As a by-product of light rubbing, structure- and air-borne sounds provide additional signals that can be used in evaluating the surface characteristics and correlating the measurements with perception [6]. Effects of some of these material and texture properties can also be measured by mounting accelerometers on a finger to detect surface roughness and slipperiness.

Similarly, accelerometers placed on a probe can provide information regarding surface roughness/smoothness and even hardness/softness of a surface through the transient oscillatory response of the probe tip when they first come into contact as discussed below. For example, surfaces that are often described as sticky correlate with the tangential force [viz., 7-9], which on an otherwise smooth surface stems from adhesive forces that are known to be dissipative and, consequently, those described as smooth and silky produce a low tangential dissipative force. The magnitudes and time (or frequency) dependence of these signals influence the perception about a surface. In addition, third bodies such as lubricants between the surfaces also affect the magnitude of the tangential dissipative force [10].
The apparatus described in this paper can measure tribological and dynamical parameters that result from friction between soft materials under very light loads, such that they can be used to relate dynamical output to material and texture properties. The simple apparatus described here mimics very light passive touch of a finger over another surface. The device has the capability to measure air- and structure-borne acoustic signals as well as the tangential contact forces and normal loads during the contact process.

\section{Test apparatus}

\subsection{Design criteria}

The functional requirements on which the design criteria are based include: (i) a finger-like mechanism that can lightly touch a moving surface, (ii) signals generated during its operation largely result from the contact of the "finger probe" with a sample, with negligible contributions of ancillary signals from other sources, and (iii) the apparatus allows access to sensors and accessories for measurements during its operation. In addition to these functional requirements, further design rules were developed for ease of operation and measurements with the apparatus.

Like a finger, the probe should be able to retract from or press on a moving surface, with a desired normal contact load similar to a light touch that an actual finger can exert. As a finger does while probing a surface, the relative motion between the probe and sample should be steady, controlled, and preferably with constant velocity. Also, the device should enable easy change of materials on either surface in order to be able to test different material combinations.

Signals generated by a light contact are normally localized and can be detected by collocated sensors. However, they are often 
a

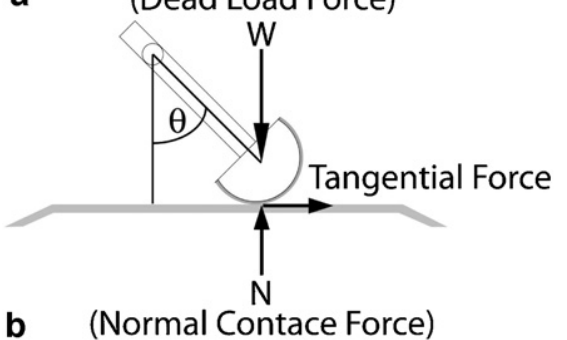

b (Normal Contace Force)

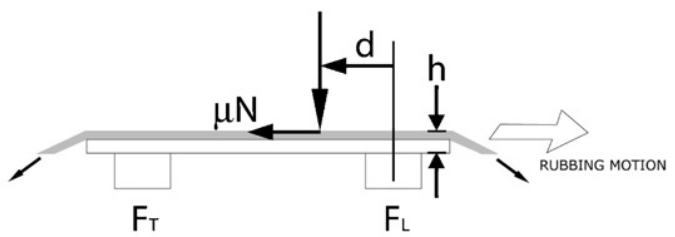

(Trailing Force) (Leading Force)

Fig. 2. (a) Contact force components between the probe and arm. (b) Schematic description of the contact force as it travels on the sample and the moment arm $h$.

contaminated by ancillary signals, such as those associated with the power and transmission components of the system, as well as sources external to the test apparatus, effects of which need to be mitigated. Just as a finger senses surface properties with the mechanoreceptors in the dermis nearest the contact area, the apparatus should be able to sense the surface topography, such as its roughness and contour, as sliding takes place. It is desirable then that the noise and vibration produced by other parts of the system than the contact area should be negligible compared with the signals to be measured. The apparatus should also have the ability

to monitor sound radiation and vibration response due to rubbing and, as importantly, have the ability to measure friction force and adhesion during contact.

\subsection{Design description}

The test apparatus, depicted in Fig. 1, consists of four subsystems. These are:

i. An arm assembly that consists of a hollow box beam, an $203.2 \mathrm{~mm}$ diameter drive pulley, motion sensor flags, bearing housing, and a floating contact platform.

ii. A finger probe assembly that consists of a finger actuator, fine and coarse height adjustments, and a parallel position adjustment mechanism for the finger.

iii. The drive train consists of a drive motor, belts and pulleys.

iv. The motion controller consists of the circuits that control the arm motion.

Each of the mechanical parts is described in the following subsections.

\subsection{The arm assembly}

The cantilever arm, constructed from a hollow box aluminum beam with rectangular cross section, rotates about a vertical post to which it is attached with high precision bearings. The arm can swing in a horizontal plane, sweeping a $90^{\circ}$ arc to slide under the stationary finger probe. Power to rotate the arm is provided by a DC-drive brush motor powered by a single voltage. A two stage belt system is used: a $12.7 \mathrm{~mm}$ timing belt transmits torque from the motor to the intermediate pulleys. The belt is arranged such that its threads face away from the center of the pulley in order

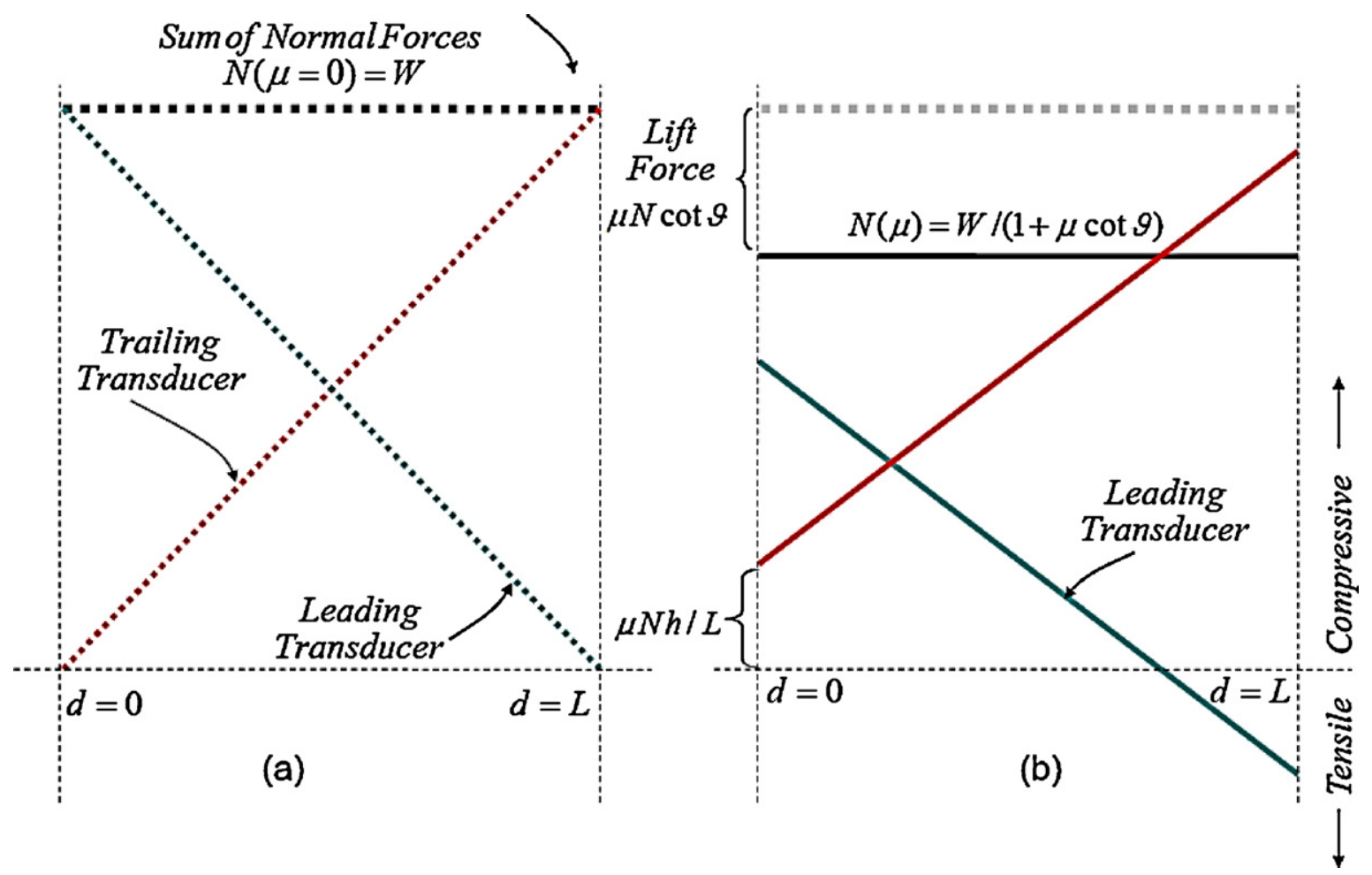

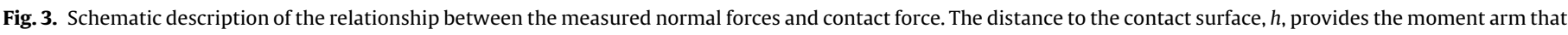

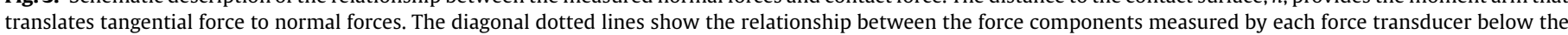

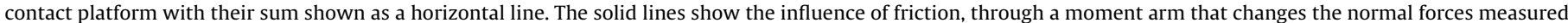

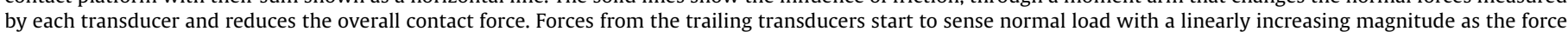
travels toward it. The leading transducer senses the full bearing of the force at the start of the contact and linearly decreases. $N=W /(1+\mu$ cot $\theta)$. 


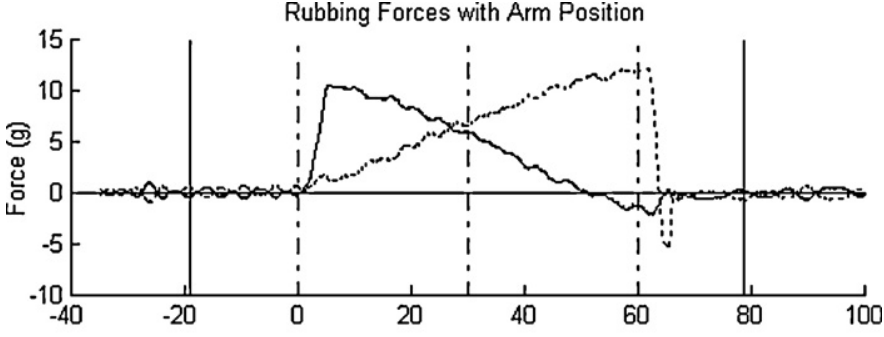

Total Normal Force

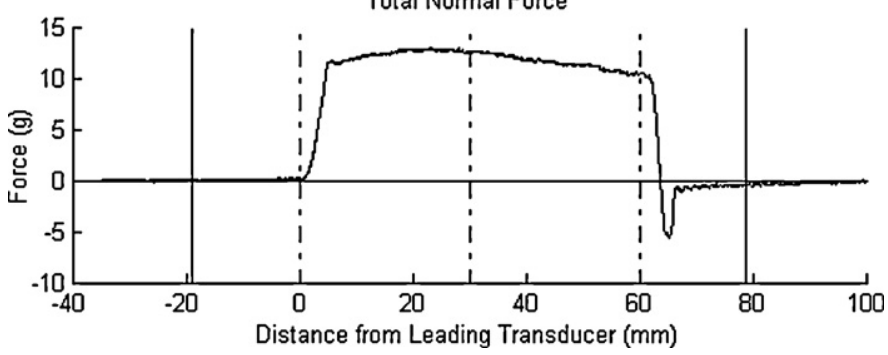

Fig. 4. A sample measurement of normal forces by the two force transducers and their sum. Artificial skin is used on the probe with PDMS as the arm material with a lubricant oil to reduce friction. The oscillations and the change in the sum throughout the contact duration result from dynamic effects of the arm.

to avoid unnecessary vibrations. The motor belt rides on a pulley that can be attached to the motor. The motor then drives the shaft through a coupling.

A $203.2 \mathrm{~mm}$ diameter pulley is used to transmit the drive torque to the arm. Placed underneath the bearing housing, this pulley is concealed by an enclosure to reduce sound transmission to the test area. The drive-belt transmits torque from the intermediate pulleys to the arm pulley. As with the motor belt, the threads of this $12.7 \mathrm{~mm}$ wide timing belt also face away from the pulley surface.

\subsection{Floating contact platform}

A floating contact platform carries the test sample on the arm and permits direct normal force measurements resulting from finger contact on the test sample. The platform consists of two $50.8 \mathrm{~mm} \times 101.6 \mathrm{~mm}$ aluminum plates mated together with
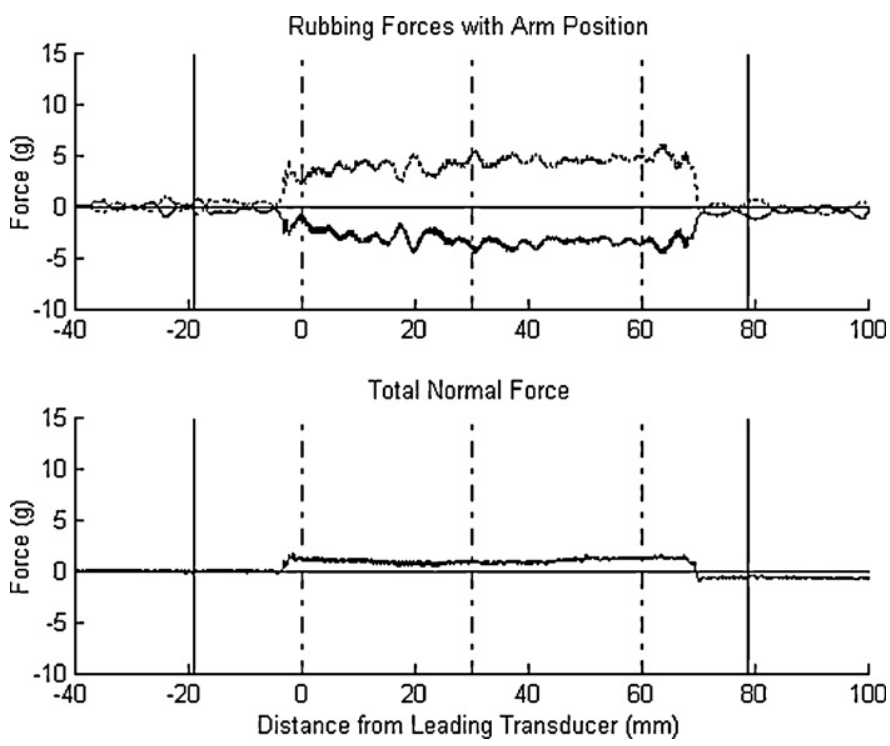

Fig. 5. Measurements with the same materials as in Fig. 4 but without oil, resulting in high friction and much lower contact force. The adhesion between the surfaces has a lifting effect.
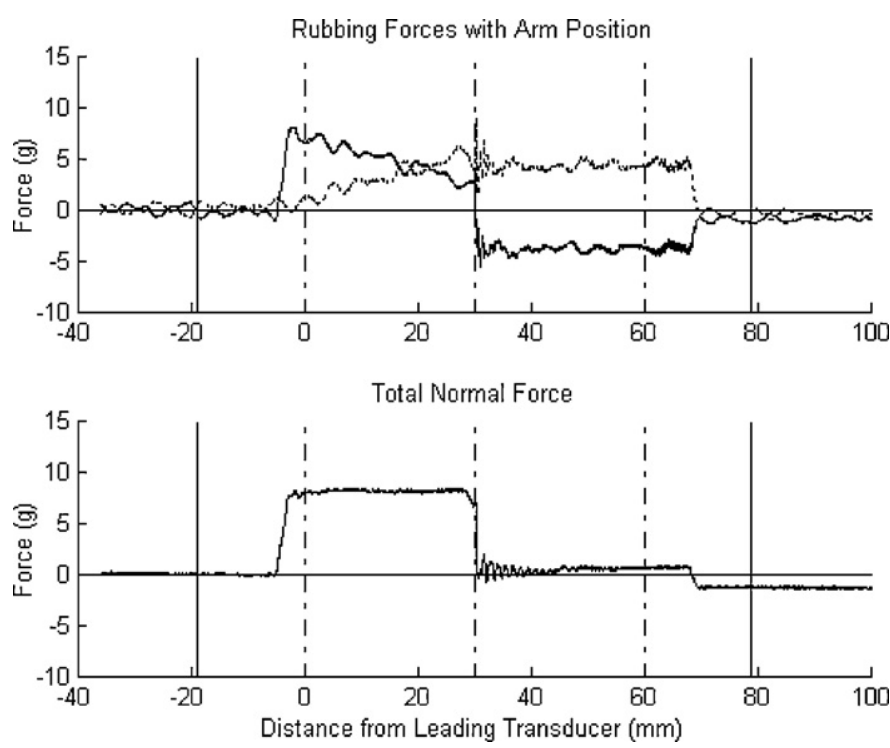

Fig. 6. A demonstration of friction effect is achieved by covering one-half of the contact surface with scotch tape, the non-adhesive side facing contact. This measurement delineates the dynamic effects caused by friction. As the probe slides over the low-friction surface to a more adhesive one, it lifts off the surface and starts to rebound as indicated by the oscillatory behavior of the force signals.

machine screws at their corners. The bottom plate is attached directly to two force transducers at its ends, illustrated schematically in Fig. 1. The top plate serves as the smooth arm surface on which the test sample is mounted, covering the mounting holes on the bottom plate. The sample materials, $25.4 \mathrm{~mm}$ wide and $100 \mathrm{~mm}$ long strips, are adhesively mounted on the top plate. With this arrangement, the plates and the sample are supported only by the two force transducers attached to the bottom wall of the hollow box beam and are otherwise isolated from the arm.

The floating platform is placed across the width and near the free end of the cantilever arm, nominally $508 \mathrm{~mm}$ from its axis of rotation, subtending an angle of $10^{\circ}$, and can accommodate different materials and surface treatments.

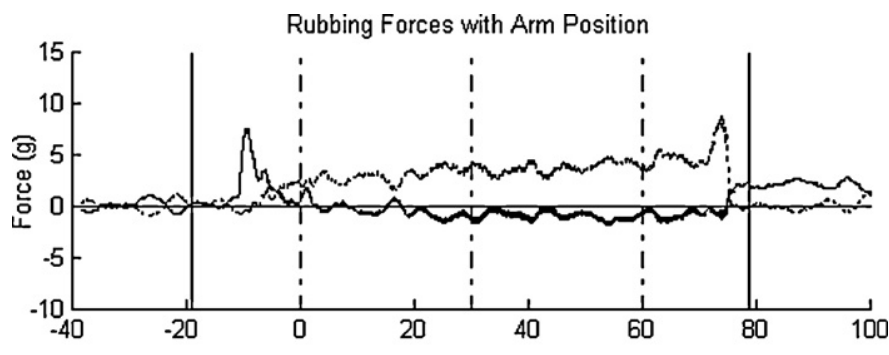

Total Normal Force

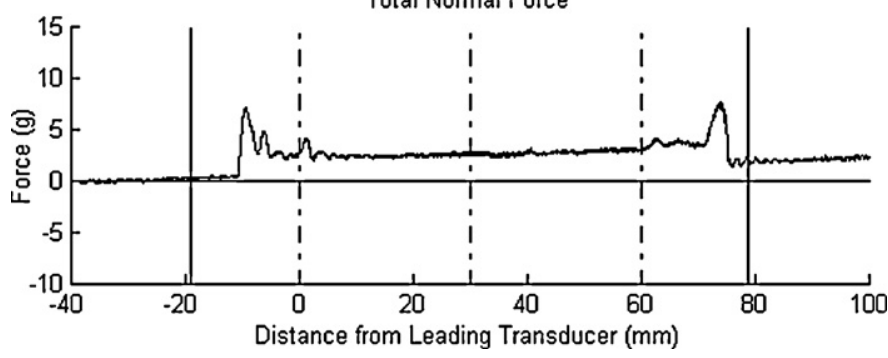

Fig. 7. Normal force measurements on a PDMS with dry smooth landings between parallel grooves. This measurement shows the rubbing of the wafer with the dry surface. Because of the smoothness of the wafer, the tangential dissipative force is large. 

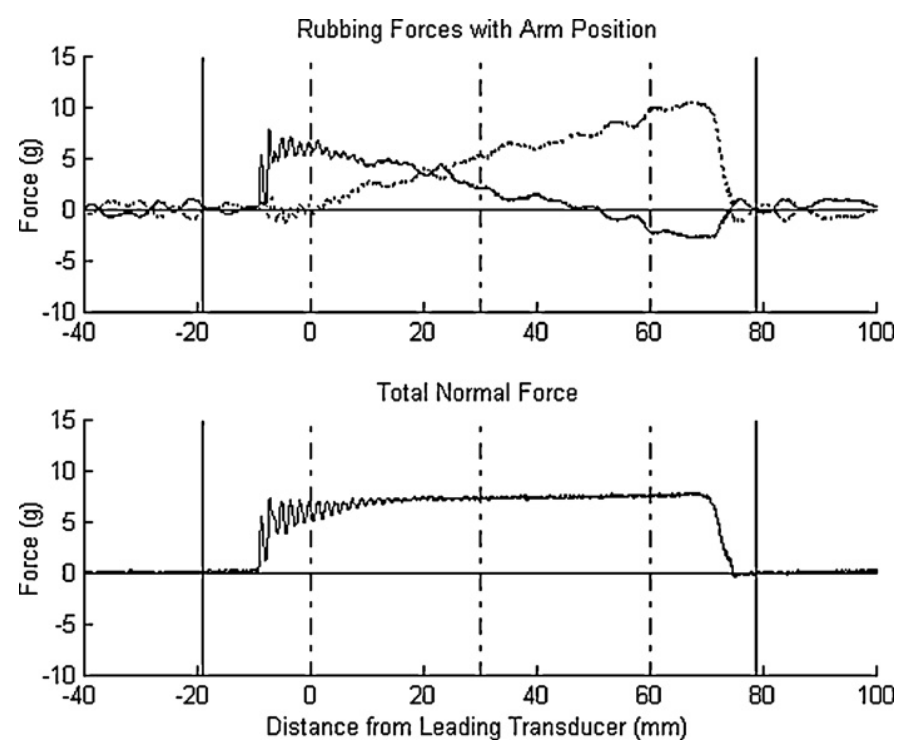

Fig. 8. Normal force measurements on a PDMS with micro-roughness induced on the surfaces between parallel grooves that reduces adhesion and increases normal contact force. The added micro-roughness reduces dissipative forces.

\subsection{Finger probe assembly}

Like a pendulum, the finger probe is designed to rotate freely about a stationary horizontal pin joint with an ultra-low friction bearing. The relative height of the pin joint is adjusted such that the axis of the finger probe makes a $45^{\circ}$ angle with the arm during contact. To assure a smooth and continuous motion, the sliding contact takes place only when the arm moves in the same direction as the angle the pendulum makes with the normal to the arm, thus resulting in tension in the axis of the finger probe and preventing development of a compressive force in its axis that would lead to ratcheting of the finger probe. To accomplish this, an embedded electromagnetic actuator at the pin joint of the finger lifts the finger probe during the arm retraction swing to avoid a collision when the direction of the arm motion is reversed. In this configuration, the finger probe is able to follow surface contours with radii larger than that of probe tip to assure continuous contact during the rubbing event.

The electromagnetic actuator at the pin joint is also used to provide a torque to the finger probe in its plane of motion in order to apply a desired force on the arm surface, on the order of $0.2-0.8 \mathrm{~N}$, similar to that exerted during actual human touch. The relative sliding speed between the finger probe and the sample on the arm is nominally $125 \mathrm{~mm} / \mathrm{s}$, representative of the probing speed of a human finger on a surface.

The tip part of the finger probe can be covered with a sleeve made of any soft test material. Both the finger material and the arm material can be changed to test different material combinations and surface treatments. Use of soft material at the tip of the finger probe provides both radial and tangential compliance. Soft material on the arm surface has compliance on normal and tangential directions.

The finger assembly is mounted on a rigid post that also has a combination of coarse and fine adjustment mechanisms to position its height. The fine adjustment is used to bring the finger to the desired height for contact with the arm material under test such that the flat mount for the normal accelerometer is parallel to the arm surface. This also assures that the finger is at a $45^{\circ}$ angle during sliding contact. The fine adjustment stage is made using a Del-Tron linear translation stage with an attached micrometer.

\subsection{Motion controller}

A custom electronics package is used to control the motion of the apparatus. The operating parameters, including the contact force and rubbing speed, are entered into the motion controller. The apparatus can then run continuously. The motion controller lifts the finger probe from the path of the arm on the return stroke.

\subsection{System dynamics}

A critical aspect of such an apparatus is to avoid ancillary vibrations and noise during the operation. Particularly for soft materials and light contact loads, any noise from the motor and the power transmission mechanism of the apparatus must be much less than the sound generated by sliding the finger over the arm.

In the case of the design described here, the test apparatus produces negligible mechanical noise compared to the acoustic emission from the contact area. For tests using very light contact forces, to further mitigate external noise problems, an acoustic enclosure was designed and constructed to enclose the test section of the arm and the finger keeping the drive mechanism outside the measurement space. Notwithstanding the low interference by the drive train, arm oscillations during measurement also need to be reduced. Reduction of vibration amplitude of the first cantilever mode of the arm was accomplished by using a $25.4 \mathrm{~mm}$ diameter stainless steel rod for the main rotational shaft of the arm as well as stiffening of both the arm and the bearing housing. With these adjustments, the first bending and torsional natural frequencies of the arm were measured as $57 \mathrm{~Hz}$ and $560 \mathrm{~Hz}$, respectively.

The following dynamic events can be sensed by the force transducers, accelerometers, and microphones: (i) Impact forces that develop during the initial contact of the finger probe with the sample on the arm, (ii) normal force exerted by the probe as it travels across the sample on the arm, (iii) impact forces that result from the rebound of probe tip following lift off the sample surface due to adhesive forces, (iv) stick-slip events as the probe slides over an adhesive surface, (v) acceleration signals representing surface roughness, and (vi) torsional oscillations of the arm excited by the stop and start as it changes direction of motion. Among these the impact force that can develop as the arm makes an initial contact with the stationary probe, case (i), is prevented by attaching a small flexible membrane with which the probe makes its initial contact and slides over it to the sample surface. Following this landing, the probe tip aligns with the arm surface thus avoiding impact excitation of the probe tip as well as additional excitation of the arm vibrations.

Instrumentation to detect these events and the measurement examples are given in the following sections.

\subsection{Instrumentation}

Two miniature accelerometers (PCB 352A24) with a sensitivity of $100 \mathrm{mV} / \mathrm{g}\left[10.2 \mathrm{mV} /\left(\mathrm{m} / \mathrm{s}^{2}\right)\right]$ and a frequency range of $1-8000 \mathrm{~Hz}$ and $0.8 \mathrm{gm}$ were used to sense the very small changes in the acceleration of the finger probe tip due to surface texture and adhesion as it travels over the arm sample. They were mounted on the two respective flat surfaces on the interior of the finger probe tip, just above the contact area, with axes parallel and normal to the arm surface, respectively, as indicated in Fig. 1. These accelerometers are AC coupled and each connected to either an Integrated Circuit Piezoelectric (ICP) power supply or to a data acquisition system that can act as an ICP device. Due to their proximity to the contact area, these accelerometers could detect vibrations induced by surface roughness.

A microphone (B\&K 4165) placed very close to the contact area, approximately $25 \mathrm{~mm}$ away from the outer surface of the finger, 


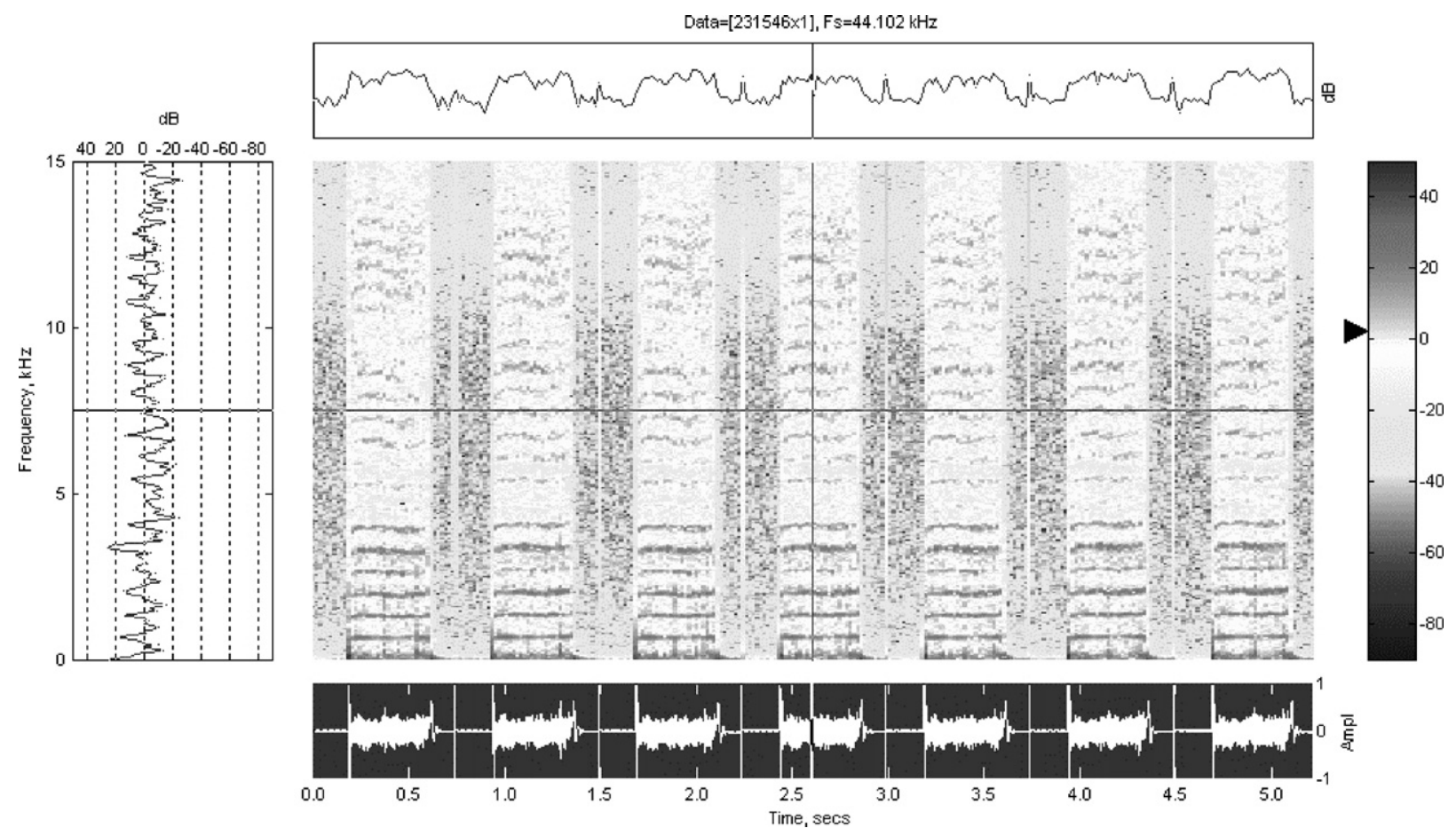

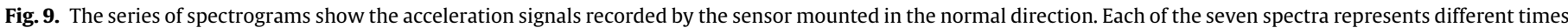
during one sliding period of the probe over the arm.

together with the necessary instrumentation, recorded the sounds generated in the contact area. The axis of the microphone is directed toward contact area.

Two piezoelectric force transducers (PCB 208C01) that support the floating contact platform at the free-end of the arm are used to measure the normal force exerted by the finger probe. Based on their configuration, the same normal force measurements also yield friction force during the rubbing events as described in the next section. To describe the operation of the system, the leading transducer refers to the transducer that is first passed over as the finger and arm come into contact. The trailing transducer refers to the transducer that is passed over before the finger terminates contact with the arm. The transducers are located inside box-channel, each mounted on an aluminum block at its bottom and connected
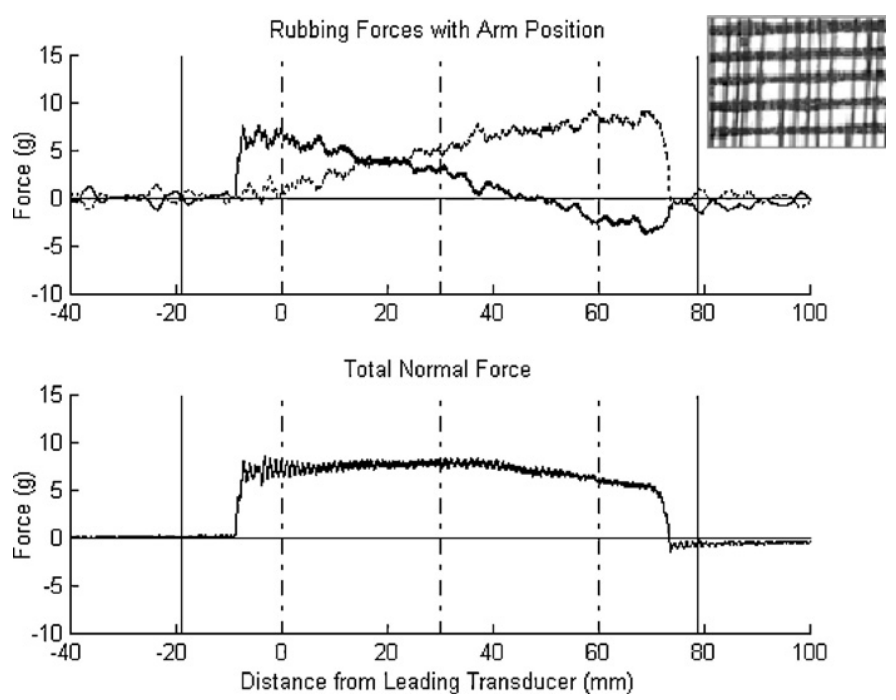

Fig. 10. This measurement shows friction forces that develop during sliding of the probe tip over silk made of synthetic silk fibers woven in a complex pattern. to the contact platform at the top. Each force transducer is connected via an ICP power supply to a data acquisition system. Their signals are DC coupled, in order to be able to measure DC loads.

It is worth noting that since these are force transducers, the voltage from the transducers will decay over time (discharge time constant $>50 \mathrm{~s}$ ) after a DC load is applied. They provide accurate measurements as long as the event is short compared to the time constant of the transducer, as in the present case. Use of load cells present an alternative sensor choice, and might be useful for much slower rubbing speeds. Although load cells greatly enhance the stability of the static load measurement, they have a lower dynamic response.

\subsection{Contact force measurement method}

A pair of identical force transducers was used to measure the normal and tangential (friction) forces during rubbing events. As the arm rotates and comes into contact with the finger probe, the force transducers mounted in the arm start to sense the force applied to the sample apportioned according to the contact position of the traveling force.

Referring to Fig. 2a, there is a kinematic relationship between the normal contact force, $N$, and the weight, or applied force, $W$ to the tip of the finger probe:

$N=\frac{W}{1+\mu \cot \theta}$

where $\theta$ is the angle the finger probe makes with the normal to the arm, in this case $45^{\circ}$, and $\mu$ refers to the coefficient of friction during contact. We note the lift force caused by friction as:

$W-N=F_{\mu} \cot \theta=\mu N \cot \theta$

The contact forces applied to the surface, the net normal force, and the moment due to friction force are apportioned to the two 


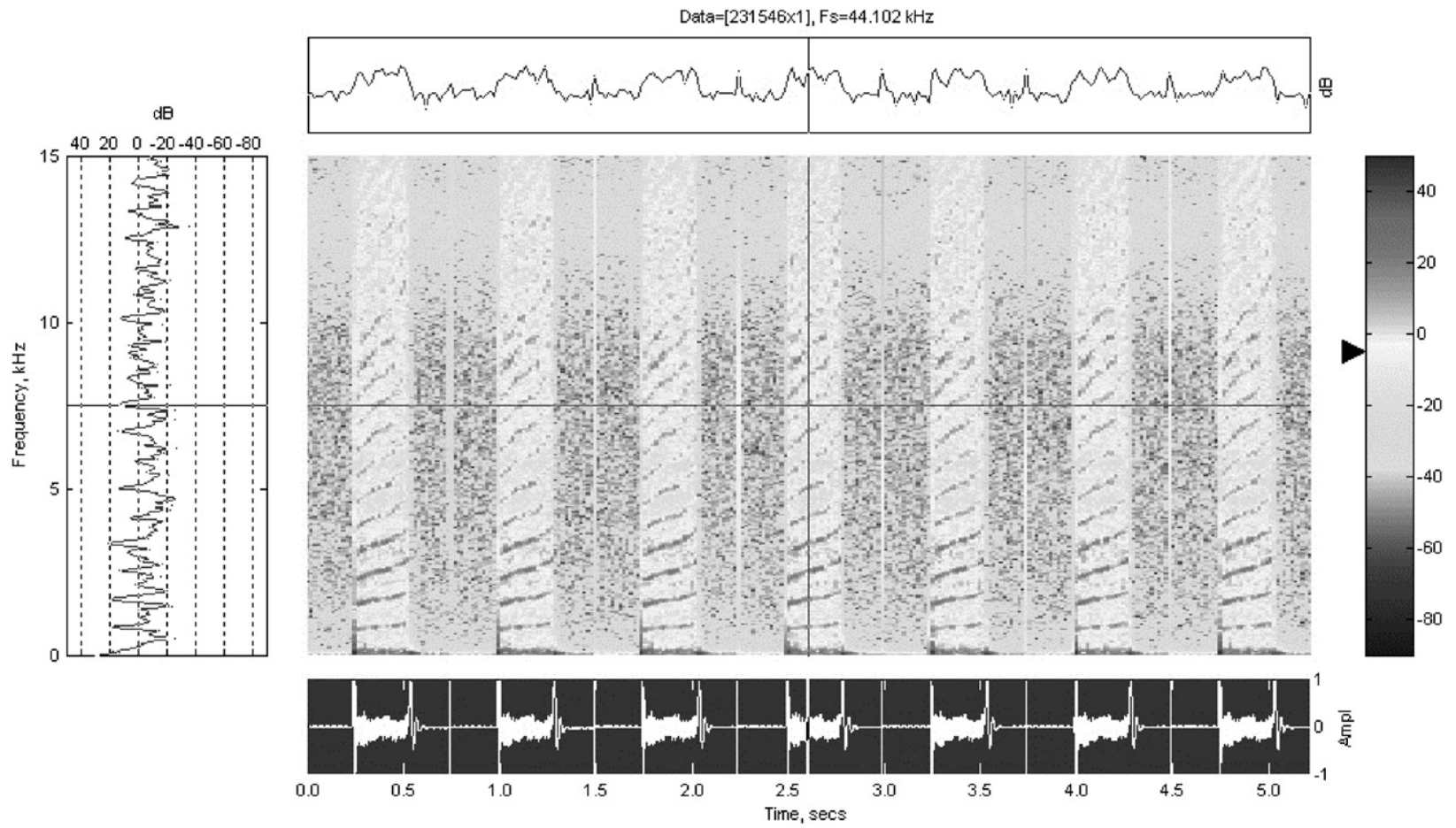

Fig. 11. Change of frequencies with speed of arm during contact as demonstrated by the slopes of the spectra and their harmonics.

supports near either end of the platform where the force transducers are placed to measure the normal forces as

$N_{L}=\frac{W}{(1+\mu \cot \theta)}\left[\frac{L-d}{L}-\mu \frac{h}{L}\right] \quad N_{T}=\frac{W}{(1+\mu \cot \theta)}\left[\frac{d}{L}+\mu \frac{h}{L}\right]$

where the subscripts $L$ and $T$ refer to the leading and trailing positions of the force transducers. As previously stated, the leading transducer is the one that is closer to the initial contact location. Since contact is made in only one direction of motion of the arm, these designations remain the same. The amplitudes of the two normal reaction forces change with distance, $d$, of the contact location from the lead transducer location. Presence of friction modifies the measured normal reaction forces by means of a moment produced by the friction force and the moment arm $h$, the distance from the transducer surface to the surface of the sample material where contact takes place, as shown in Fig. 2b.

Fig. 3 schematically demonstrates how the normal forces at the two transducer positions vary linearly as the applied contact force moves across the sample surface, with and without the presence of friction on the surface. As shown in Fig. 3a, without friction, as contact is initiated at $d=0$, the leading transducer carries the full weight of the contact load, which linearly decreases and vanishes as the contact point travels toward and reaches the trailing transducer, $d=L$. Simultaneously, the trailing transducer starts to sense a normal load as the contact point starts to travel toward it, and increases linearly to full magnitude when it reaches the trailing transducer. Their sum, shown by the horizontal dotted line, remains constant and equal to the contact load which, in the absence of friction, is equal to the applied force, $W$.

In the presence of friction, however, the slopes and the initial and final magnitudes of the diagonal lines change, as indicated by the solid lines in Fig. 3b. The lift force resulting from the moment introduced by the presence of friction reduces the initial magnitude of $N_{L}$ and increases $N_{T}$, respectively, by an amount equal to $\mu N h / L$. Their sum at any time during the sliding process equals the total normal contact force, $N$. Thus, the change in the normal contact force $N$ directly relates to the tangential, or friction, force in accordance with the inverse relationship between friction and normal force, expressed by the kinematic relation given above. In summary, friction and adhesion forces can be measured directly by measuring the normal contact forces using two force transducers as the contact force traverses over the sample.

\subsection{Measurement examples}

Typical contact force measurements, using the method described above are displayed in Figs. 4 and 5 for low- and highfriction contacts, respectively. The low-friction sample used in Fig. 4 was prepared by applying light lubricant to the normally adhesive PDMS that was used for Fig. 5. The forces measured by the leading and trailing transducers and their sum, which represents the normal contact force that directly relates to the friction (tangential contact) force, illustrate the measurement technique described above. These measurements were made using a finger probe covered with Bioskin produced by Beaulax, a skin-like polymer, sometimes referred as artificial skin. The finger rubs over the flat surface on the rotating arm as described above, which is covered by polydimethylsiloxane (PDMS). The PDMS samples were molded from flat silicon wafers. The molded samples had a smooth, sticky and soft feel to the touch. In these measurements, the transducers are positioned $60 \mathrm{~mm}$ apart and the normal load, $10.6 \mathrm{~g}$, remained the same.

As the probe traverses across the sample on the arm, the mean of the measured normal force by each transducer change linearly with distance of the contact point from force transducers, as illustrated in Fig. 3. The oscillations about the mean represent the torsional oscillations of the cantilever arm about its axis, excited by the motor-belt system, and are also observed before and after the probe travels over the sample. Because these nearly harmonic oscillations are out of phase, they cancel each other when they are summed and do not appear in the sum of the reaction forces as shown in Figs. 4 and 5.

Adhesion between the probe and the sample surface on the arm gives rise to additional dynamic phenomena. When the finger 
probe comes into contact with or as it slides over an adhesive surface, under certain conditions, the tangential adhesive force lifts the probe-tip off the arm surface. Following a brief free-flight, the probe bounces back on the surface, followed by rebounds. An example of such a rebound is observed in the force measurements shown in Fig. 6, which also gives a clear demonstration of this flutter-like phenomenon. The test sample on the arm measured to generate Fig. 6 has two regions with different surface adhesion. The leading part of the sample is covered with a smooth membrane with very low friction and the second half of the surface has highly adhesive dry PDMS. As the finger probe moves over the first half of the sample, the response is the same as that in Fig. 4, showing a relatively high normal contact force, representative of low friction. The response of the two individual transducers sums up to a smooth total normal contact force devoid of oscillations.

As the probe tip comes into contact with the adhesive part of the surface, the pendulum-like probe lifts off, its tip making an arc about its hinge, and rebounds leading to additional oscillatory signals. The mean amplitude of the total normal force is lower on this part of the sample due to the lift force caused by adhesion. The oscillations represent the pendulum rebounds of the probe. Both the magnitude of the lift and the rebound are related to, and thus contain information about, the adhesive and elastic properties of the surfaces materials.

The higher frequency oscillations observed in the second half of the sample in Fig. 6, as well as in Fig. 5, represent the local stickslip between the adhesive surface and the probe, and are absent in the measurements that involve low-friction surfaces. Similarly, the rebounds are absent in the force measurements shown in Fig. 4 whereas the measurements for the case with a strong adhesion (Fig. 5) contain strong oscillations of the normal contact forces.

In cases where the contact forces that develop due to surface roughness have much smaller amplitudes compared with the normal forces, their effects are not as easily detected by the force transducers. Instead, the two accelerometers mounted on the inside of the finger probe-tip are used to obtain spectral information about the surface characteristics. To demonstrate surface roughness effects, three silicon wafers with different surface properties were used as samples on the arm. Two of the wafers had periodic grooves cut into the surface and the third was a perfectly flat (standard) wafer. The grooves had the same trapezoidal cross section and were periodically spaced $250 \mu \mathrm{m}$ apart. Each groove was $80 \mu \mathrm{m}$ wide at the top and $58 \mu \mathrm{m}$ deep. The two wafers with the grooves were identical except for the surface roughness. One of the grooved wafers had been chemically etched so that the land (surface between the grooves) had a micro-roughness. The other wafer was perfectly flat on the lands. Each wafer could be rubbed in different directions.

Normal force measurements of the etched wafers are given in Figs. 7 and 8 for the case when the landing between the grooves is smooth and has micro-roughness, respectively. Neither case has discernable force response due to the grooves. The smooth case, Fig. 7, has high adhesion and therefore low normal contact force, similar to Fig. 5. The surface micro-roughness between the grooves, however, reduces adhesion and, as a result, has higher normal contact force. In both of these measurements, the decaying oscillations shown in the left side of the plots result from the impact of the pendulum tip with the sample on the arm at initial contact, which do not appear in Fig. 4 or 5 since the pendulum was afforded a soft landing as discussed above.

Roughness effects from the periodically spaced grooves can be seen in the acceleration response of the pendulum tip. Fig. 9, for example, shows a series of normal acceleration spectrograms representing normal acceleration of the finger probe tip.

These measurements directly represent the texture of the surfaces. Each spectrogram in Fig. 9 represents the frequency content of acceleration signals at different times, corresponding to different parts of the arm material, during a single sweep. For this particular measurement the finger probe surface was covered with a soft material and the arm surface was PDMS (10:1) with parallel grooves. The frequency peaks correspond to the spacing of the grooves with a fundamental of approximately $670 \mathrm{~Hz}$ and its harmonics. The addition of the accelerometers to the apparatus allows for additional surface characteristics to be measured that cannot be discerned with the force transducers alone.

In those cases where the surface has pronounced topography, as expected, the pendulum-like finger probe will exert sufficient pulses on the sample surface to be sensed by the force transducers. One such example is given in Fig. 10 where the sample consists of a silk made of synthetic silk fibers woven in a complex pattern.

The microphone in the system can also measure periodic events during the rubbing motion. It should also be noted that as the arm comes into contact with the finger probe, depending on the applied force by the probe, the arm may initially slow down and then speed up again. The increasing slopes of the spectral lines in Fig. 11 demonstrate this speed change, which becomes more acute at higher harmonics. The harmonics in the spectra point to nonlinear nature of the interaction during sliding as well as the slight aperiodicity of the ridges on the surface. As expected, the sound pressure measurements made very near the contact area show the same trends as the acceleration signals.

\section{Discussion and concluding remarks}

The connection between texture and material of a surface and the subjective dimensions that describe perception of it is first made through the mechanoreceptors in the dermis of fingertips. Objective evaluations of the surfaces by measuring parameters that influence the corresponding subjective dimensions require accurate physical characterization of materials and surfaces. Such measurements can ultimately lead to estimation of subjective characterization of surfaces based on objective measurable parameters.

The apparatus and the measurement method described in this paper are designed to emulate the contact conditions between a finger and a soft material. The device has the capability to make simultaneous measurements of different dynamic signals generated as the finger probe lightly slides over the surface of a soft material. These include acceleration signals measured in close proximity of the contact point within the probe tip, which are caused by and thus provide information about, the surface texture. Both the accelerometer signals and the sound pressure recorded near the contact area contain frequencies far above those that can be detected by the mechanoreceptors and provide additional useful information.

The apparatus described here also has a unique capability to measure adhesion between surfaces, which directly relates to the "stickiness" of a surface. The method developed here can measure and distinguish adhesion between surfaces and overcomes the difficulties associated with measuring friction force between soft surfaces. Use of a pair of force transducers allows the measurement of both the normal contact force and the tangential dissipative force, without interfering with the sliding process. The method relies on the lift force that develops through a moment-arm, $h$, which is the distance to the sample surface above the force transducers. The desired measurement sensitivity can be achieved by adjusting $h$.

The sensitivity of the present apparatus in making air- and structure-borne acoustic measurements under light loads is enhanced in part, due to the smooth and quiet motion the arm. The system dynamics have negligible contribution to the measurements, save for the torsional oscillations that appear in the 
individual reaction force measurements but not on their sum, as described earlier. Sample measurements show how the ordinary definition of coefficient of friction gives way to consideration of friction force as a pure adhesion, with normal as well as tangential components as illustrated in Fig. 6, clearly distinguishing the two regimes that had two surface properties.

The device described here can be used to determine and correlate tribological parameters to dynamic events and eventually to subjective evaluations.

\section{Acknowledgments}

Authors acknowledge generous support of Unilever R\&D (USA). One of the authors (AA) gratefully acknowledges support by the National Science Foundation where the author AA was employed during part of the period when this research was conducted and generous support of EU Marie Curie program for subsequent research.

\section{References}

[1] S.J. Lederman, Auditory texture perception, Perception 8 (1979) 93-103.

[2] G. Lundborg, B. Rosén, S. Lindberg, Hearing as substitution for sensation: a new principle for artificial sensibility, J. Hand Surgery 24A (1999) 219-224.

[3] C. Kayser, C.I. Petkov, M. Augath, N.K. Lohothetis, Integration of touch and sound in auditory cortex, Neuron 48 (2005) 373-384.

[4] S. Guest, C. Spence, What role does multisensory integration play in the visuotactile perception of texture? Int. J. Psychophysiol. 50 (2003) 63-80.

[5] R. Newport, J.V. Hindle, S.R. Jackson, Links between vision and somatosensation: vision can improve the felt position of the unseen hand, Curr. Biol. 11 (2001) 975-980.

[6] A. Akay, Acoustics of friction, J. Acoust. Soc. Am. 111 (2002) 1525-1548.

[7] T. Yoshioka, S.J. Bensamaïa, J.C. Craig, S.S. Hsiao, Texture perception through direct and indirect touch: an analysis of perceptual space for tactile textures in two modes of exploration, Somatosens. Mot. Res. 24 (1-2) (2007) 53-70.

[8] Mark Hollins, Adam Seeger, Gabriele Pelli, Russell Taylor, Haptic perception of virtual surfaces: scaling subjective qualities and interstimulus differences, Perception 33 (2004) 1001-1019.

[9] Michel Paré, Heather Carnahan, M. Allan, Smith, Magnitude estimation of tangential force applied to the fingerpad, Exp. Brain Res. 142 (2002) 342-348.

[10] M.J. Adams, B.J. Briscoe, S.A. Johnson, Friction and lubrication of human skin, Tribol. Lett. 26 (2007) 239-253. 